\title{
A New Fifth-Order Iterative Method for Finding MultipleRoots of Nonlinear Equations
}

\author{
R. Thukral \\ Padé Research Centre, 39 Deanswood Hill, Leeds, West Yorkshire, LS17 5J S, England
}

\begin{abstract}
In this paper, we present a fifth-order method for finding multiple zeros of nonlinear equations. Per iteration, the new method requires two evaluations of functions and two of its first derivative. It is proved that the method has a convergence of order five. Finally, so me nu mericalexamp les are given to show the performance of the presented method, and compared with some known methods.
\end{abstract}

Keywords Modified Newton Method, Root-Finding, Nonlinear Equations, Multiple Roots, Order of Convergence, Efficiency Index

\section{Introduction}

Solving nonlinear equations is one of the most important problems in numerical analysis. In this paper, we consider iterative methods to find a multiple root $\alpha$ of multiplicity $m$, that is,

$$
f^{(j)}(\alpha)=0, j=0,1, \ldots, m-1 \text { and } f^{(m)}(\alpha) \neq 0,
$$

of anonlinear equation

$$
f(x)=0,
$$

where $f: I \subset \square \rightarrow \square$ is a scalar function on an open interval $I$ and it is suffic iently s mooth in the neighbourhood of $\alpha$. In recent years, some modifications of the Newton method for multiple roots have been proposed and analysed[1,3,5-7,10]. However, there are not many methods known to handle the case of multip le roots. Hence we present a fifth-order method for finding multiple zeros of a nonlinear equation and only use four evaluations of the function per iteration. In addition, the new methodhas a better efficiency index than the third and fourth order methods given in $[1,3,4,7,10]$. In view of this fact, the new method is significantly better when compared with the established methods. Consequently, we have found that the new method is efficient and robust.

The well-known Newton's method for finding multiple roots is given by

$$
x_{n+1}=x_{n}-m \frac{f\left(x_{n}\right)}{f^{\prime}\left(x_{n}\right)},
$$

* Corresponding author:

rthukral@hotmail.co.uk (R. Thukral)

Published online at http://journal.sapub.org/ajcam

Copyright (C) 2012 Scientific \& Academic Publishing. All Rights Reserved which is quadraticallyconvergent [4]. For the purpose of this paper, we use (2) to construct a new fifth-order method.

\section{Development of the Method and Convergence Analysis}

In this section we define a new fifth-order method. In order to establish the order of convergence of the new method we state the three essential definitions.

Definition 1 Let $f(x)$ be a real function with a simple root $\alpha$ and let $\left\{x_{n}\right\}$ be a sequence of real numbers that converge towards $\alpha$. The order of convergence $p$ is given by

$$
\lim _{n \rightarrow \infty} \frac{x_{n+1}-\alpha}{\left(x_{n}-\alpha\right)^{p}}=\zeta \neq 0,
$$

where $\zeta$ is the asymptotic error constant and $p \in \square^{+}$.

Definition 2 Let $\lambda$ be the number of function evaluations of the new method. The efficiency of the new method is measured by the concept of efficiency index $[2,8]$ and defined as

$$
\mu^{1 / \lambda}
$$

where $\boldsymbol{\mu}$ is the order of convergence of the method.

Definition 3 Suppose that $x_{n-1}, x_{n}$ and $x_{n+1}$ are three successive iterations closer to the root $\alpha$ of (1). Then the computational order of convergence[9] may be approximated by

$$
\mathrm{COC} \approx \frac{\ln \left|\left(x_{n+1}-\alpha\right)\left(x_{n}-\alpha\right)^{-1}\right|}{\ln \left|\left(x_{n}-\alpha\right)\left(x_{n-1}-\alpha\right)^{-1}\right|},
$$

where $n \in \square$. 
The new fifth-order method for finding multiple root of a nonlinear equation is expressed as

$$
\begin{gathered}
y_{n}=x_{n}-m \frac{f\left(x_{n}\right)}{f^{\prime}\left(x_{n}\right)}, \\
x_{n+1}=y_{n}-m\left(1+z^{2 / m}\right)\left(\frac{f\left(y_{n}\right)}{f^{\prime}\left(y_{n}\right)}\right) .
\end{gathered}
$$

Another form of the formula (7) is given by

$$
x_{n+1}=y_{n}-m\left(1-z^{2 / m}\right)^{-1}\left(\frac{f\left(y_{n}\right)}{f^{\prime}\left(y_{n}\right)}\right),
$$

where $z=f\left(y_{n}\right) f\left(x_{n}\right)^{-1}, n \in \square, x_{0}$ is the initial value and provided that the denominators of (6) and (7) are not equal to zero.

\section{Theorem 1}

Let $\alpha \in I$ be a multiple root of multiplicity $m$ of a sufficiently differentiable function $f: I \subset \square \rightarrow \square$ for an open interval $I$. If $x_{0}$ is sufficiently close to $\alpha$ then the order of convergence of the new method defined by (7) is five.

\section{Proof}

Let $\alpha$ be a multiple root of multiplicity $m$ of a sufficiently smooth function $f(x), e=x-\alpha$ and $\widehat{e}=y-\alpha$, where $y$ is defined in (6).

Using the Taylor expansion of $f(x)$ and $f(y)$ about $\alpha$, we have

$$
\begin{aligned}
f\left(x_{n}\right)= & \left(\frac{f^{(m)}(\alpha)}{m !}\right) e_{n}^{m}\left[1+c_{1} e_{n}+c_{2} e_{n}^{2}+\cdots\right], \\
f^{\prime}\left(x_{n}\right)= & \left(\frac{f^{(m)}(\alpha)}{(m-1) !}\right) e_{n}^{m-1} \times \\
& {\left[1+\left(\frac{m+1}{m}\right) c_{1} e_{n}+\left(\frac{m+2}{m}\right) c_{2} e_{n}^{2}+\cdots\right], }
\end{aligned}
$$

where $n \in \square$ and

$$
c_{k}=\frac{m ! f^{(m+k)}(\alpha)}{(m+k) ! f^{(m)}(\alpha)} .
$$

Moreover by (6), we have

$$
\begin{aligned}
y_{n} & =e_{n}-m \frac{f\left(x_{n}\right)}{f^{\prime}\left(x_{n}\right)} \\
& =\frac{c_{1}}{m} e_{n}^{2}-\frac{(m+1) c_{1}^{2}-2 m c_{2}}{m^{2}} e_{n}^{3}+\cdots
\end{aligned}
$$

The expansion of $f\left(y_{n}\right)$ and $f^{\prime}\left(y_{n}\right)$ about $\alpha$ are given by

$$
f\left(y_{n}\right)=\left(\frac{f^{(m)}(\alpha)}{m !}\right) \hat{e}_{n}^{m}\left[1+c_{1} \widehat{e}_{n}+c_{2} \widehat{e}_{n}^{2}+\cdots\right],
$$

$$
\begin{aligned}
f^{\prime}\left(y_{n}\right)= & \left(\frac{f^{(m)}(\alpha)}{(m-1) !}\right) \hat{e}_{n}^{2 m-1} \times \\
& {\left[1+\left(\frac{m+1}{m}\right) c_{1} \widehat{e}_{n}+\left(\frac{m+2}{m}\right) c_{2} \widehat{e}_{n}^{2}+\cdots\right] . }
\end{aligned}
$$

By using (9) and (13), we get

$$
\begin{aligned}
\left(\frac{f\left(y_{n}\right)}{f\left(x_{n}\right)}\right)^{m^{-1}}= & e_{n}\left(\frac{c_{1}}{m}\right)\left[1+\frac{\left(w_{1}+w_{2}\right)}{m}\right. \\
& +\frac{\left(m^{-1}-1\right)\left(w_{1}+w_{2}\right)^{2}}{2 m} \\
& \left.+\frac{\left(m^{-1}-1\right)\left(m^{-1}-2\right)\left(w_{1}+w_{2}\right)^{3}}{6 m}\right],
\end{aligned}
$$

where

$$
\begin{gathered}
w_{1}=\frac{-(m+2) c_{1}^{2}+2 m c_{2}}{c_{1}} e_{n}, \\
w_{2}=\left(2 c_{1}^{3}\right)^{-1}\left(c_{1} m^{-1}\right)\left[(1+m)^{2}(3+m) c_{1}^{4}\right. \\
-2 m\left(3+5 m+2 m^{2}\right) c_{1}^{2} c_{2} \\
\left.+4(m-1) m^{2} c_{2}^{2}+6 m^{2} c_{1} c_{3}\right] e_{n}^{2} .
\end{gathered}
$$

Since from (7) we have

$$
e_{n+1}=\widehat{e}_{n}-m\left(1+z^{2 / m}\right)\left(\frac{f\left(y_{n}\right)}{f^{\prime}\left(y_{n}\right)}\right) .
$$

Substituting appropriate expressions in (18) and after simplification we obtain the error equation

$$
e_{n+1}=c_{1}^{2}\left(\frac{m c_{1}^{2}-2 m c_{2}+3 c_{1}^{2}}{m^{4}}\right) e_{n}^{5}+\cdots
$$

The error equation (19) establishes the fifth-order convergence of the new method defined by (7).

\section{The Established Methods}

For the purpose of comparison, we consider three fourth-order methods presented recently in $[3,10]$ and the third-order method presented in[7]. Since these methods are well established, we shall state the essential expressions used in order to calculate the approximate solution of the given nonlinear equations and thus compare the effectiveness of the new fifth-ordermethod for multiple roots.

\subsection{Wu et al. Method}

In[10], Wu et al. developed a fourth-order of convergence method, since this method is well established we state the essential expressions used in the method,

$$
y_{n}=x_{n}-m\left(\frac{f\left(x_{n}\right)}{f^{\prime}\left(x_{n}\right)}\right) \text {, }
$$




$$
x_{n+1}=y_{n}-m\left(\frac{f\left(y_{n}\right)}{f^{\prime}\left(y_{n}\right)}\right),
$$

where $n \in \square, x_{0}$ is the initial value and provided that the denominators of (21)-(22) are not equal to zero.

\subsection{Li et al. Method 1}

In[3], Li et al. developed some fourth-order convergence methods. The particular method we consider in this paper is expressed by,

$$
\begin{gathered}
y_{n}=x_{n}-\left(\frac{2 m}{m+2}\right)\left(\frac{f\left(x_{n}\right)}{f^{\prime}\left(x_{n}\right)}\right), \\
z_{n}=x_{n}-2\left(\frac{m}{m+2}\right)^{m}\left(\frac{f\left(x_{n}\right)}{f^{\prime}\left(y_{n}\right)}\right), \\
x_{n+1}=x_{n}-\left(\frac{2 m}{m+2}\right)\left(\frac{f\left(x_{n}\right)}{a f^{\prime}\left(x_{n}\right)+b f^{\prime}\left(y_{n}\right)+c f^{\prime}\left(z_{n}\right)}\right),
\end{gathered}
$$

where

$$
\begin{gathered}
a=\left(\frac{m^{6}-m^{5}-14 m^{4}+12 m^{3}+48 m^{2}-80 m+32}{8 m\left(m^{3}+2 m^{2}-8 m+4\right)}\right), \\
b=\left(\frac{-m}{16}\right)\left(\frac{3 m^{4}-6 m^{3}-20 m^{2}+40 m-16}{d\left(m^{3}+2 m^{2}-8 m+4\right)}\right), \\
c=\left(\frac{1}{16}\right)\left(\frac{m^{3}\left(m^{2}-4\right)}{d\left(m^{3}+2 m^{2}-8 m+4\right)}\right), \\
d=\left(\frac{m}{m+2}\right)^{m},
\end{gathered}
$$

where $n \in \square, x_{0}$ is the initial value and provided that the denominators of (23)-(25) are not equal to zero.

\subsection{Li et al. Method 2}

The second of the fourth-order of convergence method given in[3] is given as,

$$
\begin{gathered}
y_{n}=x_{n}-\left(\frac{2 m}{m+2}\right)\left(\frac{f\left(x_{n}\right)}{f^{\prime}\left(x_{n}\right)}\right), \\
z_{n}=x_{n}-2\left(\frac{m}{m+2}\right)^{m}\left(\frac{f\left(x_{n}\right)}{f^{\prime}\left(y_{n}\right)}\right), \\
x_{n+1}=x_{n}-\left(\frac{2 m}{m+2}\right)\left(\frac{f\left(x_{n}\right)}{a f^{\prime}\left(x_{n}\right)+b f\left(y_{n}\right)+c f\left(z_{n}\right)}\right),
\end{gathered}
$$

where

$$
a=\left(\frac{m^{6}-m^{5}-14 m^{4}+12 m^{3}+48 m^{2}-80 m+32}{8 m\left(m^{3}+2 m^{2}-8 m+4\right)}\right),
$$

$$
\begin{aligned}
& b=\left(\frac{-m}{16}\right)\left(\frac{3 m^{4}-6 m^{3}-20 m^{2}+40 m-16}{d\left(m^{3}+2 m^{2}-8 m+4\right)}\right), \\
& c=\left(\frac{1}{16}\right)\left(\frac{m^{3}\left(m^{2}-4\right)}{d\left(m^{3}+2 m^{2}-8 m+4\right)}\right),
\end{aligned}
$$

where $d$ is given by (29), $n \in \square, x_{0}$ is the initial value and provided that the denominators of (30)-(32) are not equal to zero.

\subsection{Thukral Third-Order Method}

In[7], Thukral developed a third-order of convergence method; the particular expressions of the method are given as,

$$
\begin{gathered}
y_{n}=x_{n}-\left(\frac{f\left(x_{n}\right)}{f^{\prime}\left(x_{n}\right)}\right), \\
x_{n+1}=x_{n}-\left(\frac{u f\left(x_{n}\right)^{2}}{u f^{\prime}\left(x_{n}\right) f\left(x_{n}\right)-v f^{\prime}(x) f\left(y_{n}\right)}\right),
\end{gathered}
$$

where $u=(m-1)^{m-1}, \quad v=(m)^{m-1}, n \in \square, x_{0}$ is the initial value and provided that the denominators of (36)-(37) are not equal to zero.

\section{Application of the New Fifth-Order Iterative Method}

The present fifth-order method given by (7) is employed to solve nonlinear equations with multiple roots and compare with the Wu et al., two of Liet al. and Thukral methods, (22), (25), (30) and (37), respectively. To demonstrate the performance of the newfifth-order method, we use ten particular nonlinear equations. We determine the consistency and stability of results by examining the convergence of the new iterative methods. The findings are generalised by illustrating the effectiveness of the fifth-order methods for determining the multiple root of a nonlinear equation. Consequently, we give estimates of the approximate solution produced by the methods considered and list the errors obtained by each of the methods. The numerical computations listed in the tables were performed on an algebraic system called Maple. In fact, the errors displayed are of absolute value and insignificant approximations by the various methodshave been omitted in the following tables.

The new fifth-order method requires four function evaluations and has the order of convergence five. To determine the efficiency index of the new method, we shall use the definition 2. Hence, the efficiency index of the fifth-order method given by (7) is $\sqrt[4]{5} \approx 1.4953$ whereas the efficiency index of the fourth-order and third-order methods is given as $\sqrt[4]{4} \approx 1.4142$ and $\sqrt[3]{3} \approx 1.4422$ respectively. We can see that the efficiency index of the new fifth-order method is better than the other similar methods. 
The test functions and their exact root $\alpha$ are displayed in table 1. The difference between the root $\alpha$ and the approximation $x_{n}$ for test functions with initial estimate $x_{0}$, are displayed in Table 2. In fact, $x_{n}$ is calculated by using the same total number of function evaluations (TNFE) for all methods. In the calculations, 12 TNFE are used by each method. Furthermore, the computational order of convergence (COC) is displayed in Table 3.

Table 1. Test functions and their roots

\begin{tabular}{|c|c|c|c|}
\hline Functions & $\mathrm{m}$ & Roots & Initial Estimate \\
\hline$f_{1}(x)=\left(x^{3}+x+1\right)^{7}$ & $m=7$ & $\alpha=-0.68232 \ldots$ & $x_{0}=-0.8$ \\
\hline$f_{2}(x)=\left(x e^{x^{2}}-\sin (x)^{2}+3 \cos (x)+5\right)^{6}$ & $m=6$ & $\alpha=-1.207647 \ldots$ & $x_{0}=-1.2$ \\
\hline$f_{3}(x)=\left((x-1)^{10}-1\right)^{4}$ & $m=4$ & $\alpha=0$ & $x_{0}=0.01$ \\
\hline$f_{4}(x)=(\exp (x)+x-20)^{3}$ & $m=3$ & $\alpha=2.842438 \ldots$ & $x_{0}=3$ \\
\hline$f_{5}(x)=(\cos (x)+x)^{5}$ & $m=5$ & $\alpha=-0.739085 \ldots$ & $x_{0}=-1$ \\
\hline$f_{6}(x)=\left(\sin (x)^{2}-x^{2}+1\right)^{8}$ & $m=8$ & $\alpha=1.404491 \ldots$ & $x_{0}=1.5$ \\
\hline$f_{7}(x)=\left(e^{-x^{2}}-e^{x^{2}}-x^{8}+10\right)^{6}$ & $m=6$ & $\alpha=1.239417 \ldots$ & $x_{0}=1.3$ \\
\hline$f_{8}(x)=\left(x^{2}-e^{x}-3 x+2\right)^{12}$ & $m=12$ & $\alpha=0.257530 \ldots$ & $x_{0}=0.2$ \\
\hline$f_{9}(x)=\left(\tan (x)-e^{x}-1\right)^{5}$ & $m=5$ & $\alpha=1.371045 \ldots$ & $x_{0}=1.4$ \\
\hline$f_{10}(x)=\left(\ln \left(x^{2}+3 x+5\right)-2 x+7\right)^{3}$ & $m=3$ & $\alpha=5.469012 \ldots$ & $x_{0}=6$ \\
\hline
\end{tabular}

Table 2. Comparison of various it erat ive methods

\begin{tabular}{ccccccc}
\hline$f_{i}$ & $\mathbf{( 7 )}$ & $\mathbf{( 2 2 )}$ & $\mathbf{( 2 5 )}$ & $\mathbf{( 3 0 )}$ & $\mathbf{( 3 7 )}$ & (2) \\
\hline$f_{1}$ & $0.170 \mathrm{e}-114$ & $0.832 \mathrm{e}-65$ & $0.216 \mathrm{e}-63$ & $0.155 \mathrm{e}-58$ & $0.391 \mathrm{e}-31$ & $0.841 \mathrm{e}-8$ \\
\hline$f_{2}$ & $0.213 \mathrm{e}-231$ & $0.518 \mathrm{e}-124$ & $0.261 \mathrm{e}-128$ & $0.145 \mathrm{e}-121$ & $0.400 \mathrm{e}-62$ & $0.204 \mathrm{e}-15$ \\
\hline$f_{3}$ & $0.577 \mathrm{e}-153$ & $0.453 \mathrm{e}-86$ & $0.329 \mathrm{e}-86$ & $0.306 \mathrm{e}-79$ & $0.193 \mathrm{e}-41$ & $0.432 \mathrm{e}-11$ \\
\hline$f_{4}$ & $0.591 \mathrm{e}-130$ & $0.310 \mathrm{e}-72$ & $0.219 \mathrm{e}-75$ & $0.117 \mathrm{e}-67$ & $0.151 \mathrm{e}-35$ & $0.570 \mathrm{e}-2$ \\
\hline$f_{5}$ & $0.515 \mathrm{e}-137$ & $0.180 \mathrm{e}-82$ & $0.129 \mathrm{e}-67$ & $0.834 \mathrm{e}-64$ & $0.341 \mathrm{e}-29$ & $0.120 \mathrm{e}-3$ \\
\hline$f_{6}$ & $0.103 \mathrm{e}-127$ & $0.182 \mathrm{e}-73$ & $0.128 \mathrm{e}-66$ & $0.708 \mathrm{e}-63$ & $0.117 \mathrm{e}-31$ & $0.205 \mathrm{e}-2$ \\
\hline$f_{7}$ & $0.181 \mathrm{e}-94$ & $0.304 \mathrm{e}-54$ & $0.526 \mathrm{e}-54$ & $0.720 \mathrm{e}-49$ & $0.133 \mathrm{e}-27$ & $0.687 \mathrm{e}-7$ \\
\hline$f_{8}$ & $0.207 \mathrm{e}-252$ & $0.356 \mathrm{e}-143$ & $0.426 \mathrm{e}-113$ & $0.797 \mathrm{e}-112$ & $0.340 \mathrm{e}-51$ & $0.933 \mathrm{e}-17$ \\
\hline$f_{9}$ & $0.453 \mathrm{e}-90$ & $0.764 \mathrm{e}-51$ & $0.259 \mathrm{e}-55$ & $0.473 \mathrm{e}-56$ & $0.133 \mathrm{e}-30$ & $0.882 \mathrm{e}-7$ \\
\hline$f_{10}$ & $0.186 \mathrm{e}-244$ & $0.197 \mathrm{e}-144$ & $0.443 \mathrm{e}-109$ & $0.110 \mathrm{e}-107$ & $0.931 \mathrm{e}-49$ & $0.132 \mathrm{e}-1$ \\
\hline
\end{tabular}


Table 3. COC of various iterative methods

\begin{tabular}{ccccccc}
\hline$f_{i}$ & $\mathbf{( 7 )}$ & $\mathbf{( 2 2 )}$ & $\mathbf{( 2 5 )}$ & $\mathbf{( 3 0 )}$ & $\mathbf{( 3 7 )}$ & $\mathbf{( 2 )}$ \\
\hline$f_{1}$ & 5.0001 & 4.0001 & 3.9999 & 4.0000 & 3.0001 & 1.9983 \\
\hline$f_{2}$ & 5.0000 & 4.0000 & 4.0000 & 4.0000 & 3.0000 & 2.0000 \\
\hline$f_{3}$ & 5.0001 & 5.3738 & 3.9999 & 3.9999 & 3.0000 & 1.9997 \\
\hline$f_{4}$ & 5.0000 & 4.0000 & 4.0002 & 4.0000 & 3.0001 & - \\
\hline$f_{5}$ & 5.0000 & 4.0001 & 4.0001 & 4.0001 & 2.9999 & - \\
\hline$f_{6}$ & 4.9999 & 4.0002 & 4.0000 & 4.0001 & 3.0000 & - \\
\hline$f_{7}$ & 4.9999 & 4.0000 & 4.0000 & 4.0000 & 3.0000 & 1.9961 \\
\hline$f_{8}$ & 4.9954 & 3.9999 & 4.0000 & 4.0001 & 2.9727 & 2.0000 \\
\hline$f_{9}$ & 5.0000 & 4.0000 & 3.9999 & 3.3270 & 3.0001 & 1.9984 \\
\hline$f_{10}$ & 5.0000 & 4.0000 & 4.0000 & 4.0001 & 3.0000 & - \\
\hline
\end{tabular}

\section{Conclusions}

In this paper, we have demonstrated the performance of a new fifth-order method for solving nonlinear equations with multiple roots. Convergence analysis proves that the newmethod preserves its order of convergence.Furthermore, we have examined the effectiveness of the new fifth-order iterative method by showing the accuracy of the multiple roots of several nonlinear equations. The main purpose of demonstrating the newmethod fordifferent types of nonlinear equations was purely to illustrate the accuracy of the approximate solution and the computational order of convergence. Finally, the advantages of the new iterative methods are; it is simple to compute, does not contain any long expressions of $m$, (see Li et al methods), has a better efficiency index than the methods considered and hence it may be considered a very good alternative to the classical methods.

\section{REFERENCES}

[1] C. Chun, B. Neta, A third-order modification of Newton's method for multiple roots, Appl. Math. Comput. 211 (2009) 474-479.
[2] W. Gautschi, Numerical Analysis: an Introduction, Birkhauser, 1997.

[3] S. G. Li, L. Z. Cheng, B. Neta, Some fourth-order nonlinear solvers with closed formulae for multiple roots, Comp. Math. Appl. 58 (2010) 126-135.

[4] E. Schroder, Uber unendich viele Algorithmen zur Auflosung der Gleichungen, Math. Ann. 2 (1870) 317-365.

[5] J. R. Sharma, R. Sharma, Modified Jarratt method for computing multiple roots, Appl. Math. Comput. 217 (2010) 878-881.

[6] L. Shengguo, L. Xiangke, C. Lizhi, A new fourth-order iterative method for finding multiple roots of nonlinear equations, Appl. Math. Comput. 215 (2009) 1288-1292.

[7] R. Thukral, A new third-order iterative method for solving nonlinear equations with multiple roots, J. Math. Comput. 6 (2010) 61-68.

[8] J. F. Traub, Iterative Methods for solution of equations, Chelsea publishing company, New York 1977.

[9] S. Weerakoon, T. G. I. Fernando, A variant of Newton's method with acceler ated third-order convergence, Appl. Math. Lett. 13 (2000) 87-93.

[10] Z. Wu, X. Li, A fourth-order modification of Newton's method for multiple roots, IJRRAS 10 (2) (2012) 166-170. 